\title{
Characterization of Temperature Field Distribution in Large-Size Paratellurite Crystals Applied in Acousto-Optic Devices
}

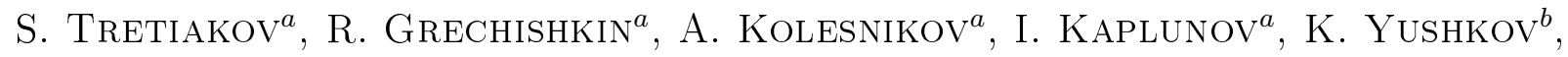 \\ V. MOLChANOV ${ }^{b}$ AND B.B.J. LindE ${ }^{c}$ \\ ${ }^{a}$ Tver State University, 170100 Russia \\ ${ }^{b}$ National University of Science and Technology "MISIS", 119049 Moscow, Russia \\ ${ }^{c}$ Institute of Experimental Physics, University of Gdańsk, 80-952 Gdańsk, Poland
}

\begin{abstract}
Temporal and spatial distribution of temperature field was studied in operating acousto-optic devices on base of large-size paratellurite crystals. The study was carried out in real time by means of thermal imaging technique. Dynamics of heat release in a hot deflector and a hot tunable filter was examined at different frequencies and driving electric power levels applied to piezoelectric transducers. The study was also based on measurements of standing wave ratio and analysis of the Smith charts.
\end{abstract}

DOI: $10.12693 /$ APhysPolA.127.72

PACS: 42.70.-a, 42.79.-e, 65.40.-b, 07.20.-n

\section{Introduction}

Heat release in an operating $(\mathrm{AO})$ device is inevitably accompanied by a change of physical properties of materials used, which means a change of the acousto-optic parameters [1-3]. Paratellurite crystals $\left(\alpha-\mathrm{TeO}_{2}\right)$ are among the most effective and called-for $\mathrm{AO}$ materials for visible and medium-infrared wavebands which explains the interest to this topic in a number of studies [4-9]. The paratellurite material refractive indexes for ordinary $n_{\mathrm{o}}$ and extraordinary $n_{\mathrm{e}}$ beams most important in acousto-optics are characterized (at the wavelength of $\lambda=1.06 \mu \mathrm{m}$ ) by the following temperature dependences $[3,5]: n_{\mathrm{o}}(T)=2.20386+7.2 \times 10^{-6} T$ and $n_{\mathrm{e}}(T)=2.34792+3.9 \times 10^{-6} T$. The coefficient of volume expansion according to [10] is $\alpha=4 \times 10^{-5} \mathrm{~K}^{-1}$. In operating regime of the practical $\mathrm{AO}$ devices under study the sound wave propagates at an angle of $3.35^{\circ}$ to the crystal direction [110]. For this case the temperature dependence of the sound velocity is described as $V(T)=6.555 \times 10^{4}+7.67 T \mathrm{~cm} \mathrm{~s}^{-1}$, where $V_{s}=657 \mathrm{~m} \mathrm{~s}^{-1}$ at $T=20^{\circ} \mathrm{C}[5]$.

According to [10] the constants of elastic stiffness $c_{i k}$ decrease with the increase of the temperature, whereas the effective constant $\left(c_{11}-c_{12}\right) / 2$ increases, and finally the coefficient of acousto-optic quality $M_{2}$ decreases with temperature by about $4 \times 10^{-17} \mathrm{~s}^{3} \mathrm{~g}^{-1}$ in the temperature interval of $20-60^{\circ} \mathrm{C}$ (for $V_{s}$ direction [110]). It should be noted that at different temperatures the experimental measurements of the AO figure of merit may be different from that predicted by the formula $M_{2}=n^{6} p^{2} / \rho V^{3}$, where $p$ is the photoelastic constant and $\rho$ is the density, even if the temperature dependence of the four values are known with high accuracy. Firstly, the increase of the temperature should have an effect on the physical properties of the contact between the piezotransducer and the crystal. Next, the temperature rise alters state of structure defects having influence on the propagation of light and acoustic waves. Calculation of the temperature field of the acoustic column is a complicated problem because of high anisotropy of the thermal conductivity of paratellurite and dissimilar constructive parts surrounding the working element. The dynamics of the temperature fields in paratellurite has specific features due to extremely small thermal conductivity $\lambda \approx 10^{5} \mathrm{erg} \mathrm{cm}^{-1} \mathrm{~K}^{-1} \mathrm{~s}^{-1}[10]$ as compared to majority of other acousto-optic materials. This factor tends to slow down the processes of smoothing the temperature profile with a change of acoustic power. In this respect a direct observation of the temperature distribution in acousto-optic lines will be especially useful for the experimental study of multi-factor heat releasing processes in acousto-optic devices working in various regimes.

In the present work we focus on the peculiarities of the temperature distribution in large-size $\left(36 \times 25 \times 22 \mathrm{~mm}^{3}\right)$ acousto-optic line on the base of paratellurite single crystal exploited as a part of tunable acousto-optic filter in a wide range of ultrasound excitation $(50-250 \mathrm{MHz})$ and electric power applied to the piezoelectric transducer $(0.5-3.5 \mathrm{~W})$. The filter was intended for astrophysical spectral studies and was described in detail elsewhere [11, 12]. Large sizes of acousto-optic crystals necessary for high spectral resolution imposes strict requirements on thermal stabilization and temperature drift compensation of filter parameters already used in terrestrial telescopes and to be used in space applications.

\section{Experimental}

In this study we used an infrared thermovision FLIR 250T camera to perform the temperature tests. The camera was installed in front of the device and focused on it so that the temperature field distribution was observed in the plane of the projection of the sound column on the output crystal face. 
The tests were performed for an AO tunable filter based on a crystal $\mathrm{TeO}_{2}$ element having the dimension $36 \times 25 \times 22 \mathrm{~mm}^{3}$ (Fig. 1a). In addition, for the sake of comparison a deflector was fabricated in a medium-size crystal $\left(17 \times 15 \times 12 \mathrm{~mm}^{3}\right.$, volume $3.06 \mathrm{~cm}^{3}$, smaller than that of the filter by a factor of 6.5) (Fig. 1b).

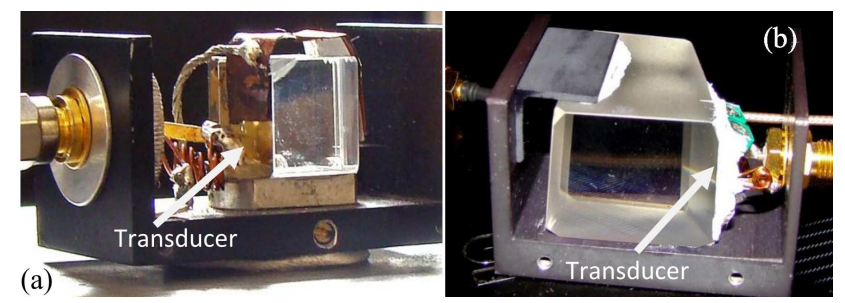

Fig. 1. (a) Acousto-optic deflector and (b) tunable filter on the base of paratellurite crystals under testing (cover removed).

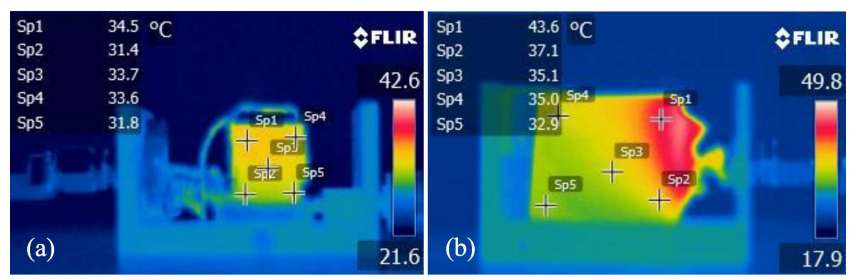

Fig. 2. Thermal images of the acousto-optic deflector (a) and tunable filter (b). Cross marks correspond to measurement points Sp1-Sp5.

Five points of observation, Sp1-Sp5, were allocated in the center and at the periphery of the thermal images as shown in Fig. 2 in order to obtain information on the temperature distribution over the output face.

\section{Results}

Figure 3 shows time dependence of the temperature measured at the reference points Sp1-Sp5 of the deflector crystal.
In order to clarify the correlation between the heat release intensity and excitation frequency, the full impedance Smith charts and frequency dependence of the SWR were measured (Fig. 4).

Figure 5 shows time dependence of the temperature of the AO filter element while Fig. 6 illustrates the Smith charts and frequency dependence of the SWR.
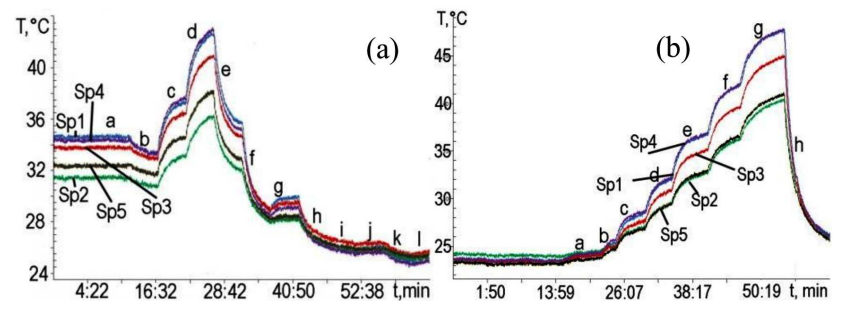

Fig. 3. (a) Time and frequency dependence of the temperature at reference points Sp1-Sp5 of the deflector crystal registered at a speed of 25 frames $/ \mathrm{s}$ with $10 \mathrm{MHz}$ increments from 50 to $160 \mathrm{MHz}(a, b, \ldots, l)$ each $300 \mathrm{~s}$ and constant power; (b) temperature changes at the Sp1-Sp5 observation points for different power values changed stepwise by $0.5 \mathrm{~W}$ increments and starting from $0.5 \mathrm{~W}$ (region (a), $1.0 \mathrm{~W}$ (b), $1.5 \mathrm{~W}$ (c), .. etc. Generator frequency $80 \mathrm{MHz}$ (b).
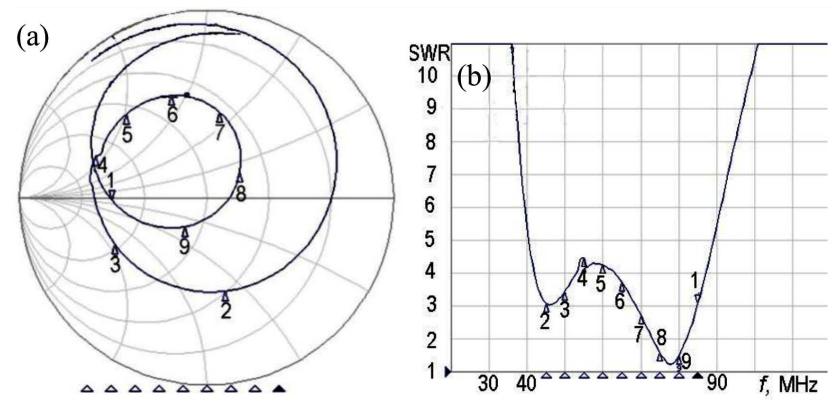

Fig. 4. Smith impedance diagram (a) and SWR frequency dependence of the $\mathrm{AO}$ deflector (b).
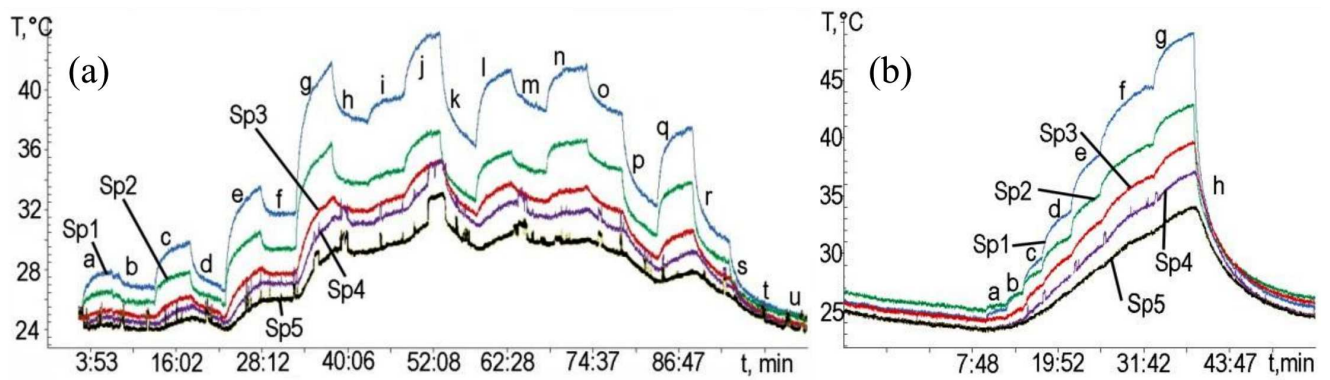

Fig. 5. (a) Time and frequency dependence of the temperature at reference points Sp1-Sp5 of the AO filter crystal registered at a speed of 25 frames/s with $10 \mathrm{MHz}$ increments from 50 to $250 \mathrm{MHz}(a, b, \ldots, u)$ each $300 \mathrm{~s}$ and constant power; (b) temperature changes at the Sp1-Sp5 observation points for different power values changed stepwise by $0.5 \mathrm{~W}$ increments starting from $0.5 \mathrm{~W}$ (region (a), 1.0 W (b), $1.5 \mathrm{~W}$ (c), .. etc. Generator frequency $140 \mathrm{MHz}$ (b).

\section{Discussion}

Analysis of the dependence on time of the heating and cooling elements of the AO devices shows that in the
AO filter sample (Fig. 3), the dynamics of the heating and cooling is several times lower than that in the AO deflector (Fig. 5). 

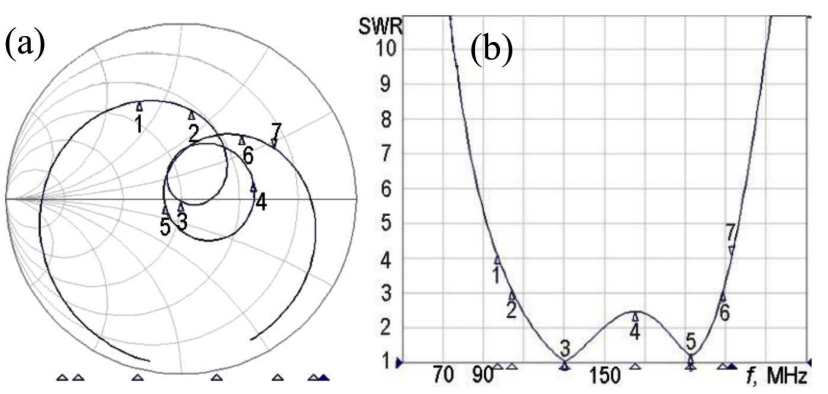

Fig. 6. (a) Smith impedance chart and (b) SWR frequency dependence of the AO filter.

At the point $\mathrm{Sp} 1$ of the filter crystal which is closest to the piezoelectric transducer the increase of the power by $0.5 \mathrm{~W}$ results in a heating at the rate of $0.8^{\circ} \mathrm{C} / \mathrm{min}$. At the outermost point $\mathrm{Sp} 5$ the rate is $0.4^{\circ} \mathrm{C} / \mathrm{min}$. Switching off of the device results in cooling rates of 6 and $2.5^{\circ} \mathrm{C} / \mathrm{min}$ at points $\mathrm{Sp} 1$ and $\mathrm{Sp} 5$, respectively.

For the deflector the corresponding values of the heating rates are 2.4 and $1.4^{\circ} \mathrm{C} / \mathrm{min}$ at $\mathrm{Sp} 1$ and $\mathrm{Sp} 5$, respectively, and 25 and $10^{\circ} \mathrm{C} / \mathrm{min}$ for the same points on cooling.

Maximal temperature gradient values in both types of the $\mathrm{AO}$ devices are close to each other and are 4.2 and $4.7 \mathrm{~K} / \mathrm{cm}$ for the filter and deflector, respectively. It is evident that crystal heating will be the larger the higher is the energy output of the transducer, i.e. theoretically at standing wave ratio $(\mathrm{SWR})=1$. This correlation is in good agreement with the experimental observations.

It follows from Fig. 5 that the temperature maxima are attained at constant power at the frequencies of 110 , 140 and $190 \mathrm{MHz}$. At the same time the SWR for these frequencies according to the data presented in Fig. $6 \mathrm{~b}$ are, correspondingly, 2.1, 1.25, and 1.2. These frequencies are very close to the SWR minima for the filter at the point Sp3 (130.4 MHz, SWR = 1.03), and Sp5 (192.7 MHz, SWR $=1.22)$. These points correspond to the central region of the Smith chart (Fig. 6a).

Maximal temperatures of the deflector crystal are attained at constant power at 75 and $80 \mathrm{MHz}$. These frequencies are situated close to the absolute SWR minimum between points 8 and 9 in Fig. 4, corresponding to 75 and $80 \mathrm{MHz}$ for which SWR is equal to 1.58 and 1.48 . These points belong to the central region of the Smith chart (Fig. 4a).

\section{Conclusion}

In summary, we performed real-time observations of the spatial and temporal temperature distribution during operation of two types of AO devices using bulk acoustooptic $\mathrm{TeO}_{2}$ crystals. It is shown that due to low heat conductivity of $\mathrm{TeO}_{2}$ rather large temperature gradients of $4-5 \mathrm{~K} / \mathrm{cm}$ may arise during normal operation regimes. This effect is pronounced the more the smaller is the SWR value. The temperature gradients in the crystal lead to an additional distortion of acoustic and light wave fronts having a deterioration effect on the transmission function of AO devices. This important factor should be taken into account in the design of high-performance AO instruments.

\section{Acknowledgments}

The work was carried out with financial support in part from the Ministry of Education and Science of the Russian Federation in the framework of Program NUST "MISiS"(No. K1-2014-008), RFBR Research Project No. 1407-0088 A and Federal Target Program "Research and Development in the Priority Directions of Scientific Engineering Complex of Russia for 2014-2020", Agreement 14.574.21.0113.

\section{References}

[1] V.B. Voloshinov, K.B. Yushkov, B.B.J. Linde, J. Opt. A Pure Appl. Opt. 9, 341 (2007).

[2] B.B.J. Linde, J. Mol. Quant. Acoust. 27, 169 (2006).

[3] V.Ya. Molchanov, K.B. Yushkov, B.B.J. Linde, J. Paczkowski, N. Ponikwicki, AIP Conf. Proc.-Am. Inst. Phys. 1433, 72 (2012).

[4] R.R. Rice, Appl. Opt. 17, 1824 (1978).

[5] V. Balakshy, V. Voloshinov, V. Karasev, V. Molchanov, V. Semenkov, Proc. SPIE 2713, 164 (1996).

[6] P. Maák, T. Takács, A. Barócsi, E. Kollár, V. Szekely, P. Richter, Opt. Commun. 266, 419 (2006).

[7] G. Mihajlik, P. Maak, A. Barocsi, P. Richter, Opt. Commun. 282, 1961 (2009).

[8] P. Maák, T. Takács, A. Barócsi, E. Kollár, P. Richter, Ultrasonics 51, 441 (2011).

[9] R. Jiang, Z. Zhou, X. Ly, Z. Shogun, H. Zhifeng, H. Zhou, Ultrasonics 52, 643 (2012).

[10] J. Ohmachi, N. Uchida, J. Appl. Phys. 41, 2307 (1970).

[11] V.Ya. Molchanov, O.Yu. Makarov, A.I. Kolesnikov, Techn. Digest 23, AAO-01 (2001).

[12] V.Ya. Molchanov, V.M. Lyuty, V.F. Esipov, S.P. Anikin, O.Yu. Makarov, N.P. Solodovnikov, Astron. Lett. 28, 713 (2002). 